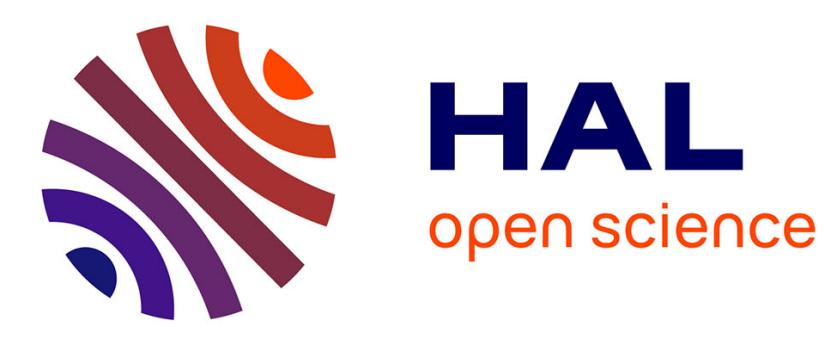

\title{
Achievability of Efficient Satisfaction Equilibria in Self-Configuring Networks
}

François Mériaux, Samir Perlaza, Samson Lasaulce, Zhu Han, Vincent Poor

\section{To cite this version:}

François Mériaux, Samir Perlaza, Samson Lasaulce, Zhu Han, Vincent Poor. Achievability of Efficient Satisfaction Equilibria in Self-Configuring Networks. International Conference on Game Theory for Networks (Gamenets), May 2012, Vancouver, Canada. pp.1-15. hal-00782498

\section{HAL Id: hal-00782498 https://hal.science/hal-00782498}

Submitted on 29 Jan 2013

HAL is a multi-disciplinary open access archive for the deposit and dissemination of scientific research documents, whether they are published or not. The documents may come from teaching and research institutions in France or abroad, or from public or private research centers.
L'archive ouverte pluridisciplinaire HAL, est destinée au dépôt et à la diffusion de documents scientifiques de niveau recherche, publiés ou non, émanant des établissements d'enseignement et de recherche français ou étrangers, des laboratoires publics ou privés. 


\title{
Achievability of Efficient Satisfaction Equilibria in Self-Configuring Networks
}

\author{
F. Mériaux ${ }^{\star}$, S. M. Perlaza ${ }^{\dagger}$, S. Lasaulce ${ }^{\star}$, Z. $\operatorname{Han}^{+}$, and H. V. Poor ${ }^{\dagger}$ \\ * Laboratoire des Signaux et Systèmes - LSS (CNRS-SUPELEC-Paris Sud), \\ Gif-sur-Yvette, 91192 France. \\ $\dagger$ Department of Electrical Engineering, Princeton University, \\ Princeton, NJ 08542 USA \\ + Department of Electrical and Computer Engineering, University of Houston, \\ Houston, TX 77004 USA
}

Key words: Supermodular games, Power control, Efficient Satisfaction Equilibrium, Games in Satisfaction Form.

\begin{abstract}
In this paper, a behavioral rule that allows radio devices to achieve an efficient satisfaction equilibrium (ESE) in fully decentralized self-configuring networks (DSCNs) is presented. The relevance of ESE in the context of DSCNs is that at such state, radio devices adopt a transmission/receive configuration such that they are able to simultaneously satisfy their individual quality-of-service (QoS) constraints. An ESE is also an efficient network configuration, i.e., individual QoS satisfaction is achieved by investing the lowest possible effort. Here, the notion of effort refers to a preference each radio device independently establishes among its own set of actions. In particular, the proposed behavioral rule requires less information than existing rules, as in the case of the classical best response dynamics and its variants. Sufficient conditions for convergence are presented in a general framework. Numerical results are provided in the context of a particular uplink power control scenario, and convergence from any initial action profile to an ESE is formally proved in this scenario. This property ensures the proposed rule to be robust to the dynamic arrival or departure of radio devices in the network.
\end{abstract}

\section{Introduction}

A decentralized self-configuring network (DSCN) is basically an infrastructureless communication system in which radio devices autonomously choose their own transmit/receive configuration in order to guarantee reliable communication. In particular, a transmit/receive configuration can be described in terms of power allocation polices, coding-modulation schemes, scheduling policies, decoding order, etc. Typical examples of DSCNs are wireless sensor networks, short range networks in the ISM bands (e.g., Wi-Fi, Bluetooth, ZigBee, etc,), femto-cell 
networks (e.g., femto cells in LTE-A) and adhoc networks in general. The underlying feature of DSCNs is that transmitters directly communicate with their respective receivers without the intervention of a central controller. Thus, the main limitation of these networks to actually provide QoS is the mutual interference arising from the uncoordinated interaction of radio devices subject to mutual interference. Within this context, the notion of QoS provisioning translates into the need for designing behavioral rules such that radio devices autonomously adapt their transmission configurations in order to meet the minimum requirements for their communications to take place satisfactorily. In particular, similar reconfigurable capabilities have been already mentioned in [6] in the context of cognitive radios.

In general, the decentralized nature of the QoS provisioning task in DSCN has fostered the use of tools from game theory (GT) [7, 5], strategic learning theory [9], distributed optimization and variational inequality theory [11] to the analysis of QoS provisioning in this scenario. In this paper, we focus on a particular formulation of the QoS provisioning problem, namely games in satisfaction form [8]. More specifically, we provide behavioral rules that allow radio devices to achieve an efficient satisfaction equilibrium (ESE) in DSCNs. The notion of ESE, as introduced in [8], refers to a network state in which all the network devices are able to satisfy their individual QoS constraints by investing the minimum effort. Often, we associate the notion of high effort with transmit/receive configurations that might represent an additional waste of energy to satisfy the individual QoS constraints. In this context, one of the main contributions of this paper is the introduction of a behavioral rule that allows the network to achieve an ESE using only local information. Another important contribution is a set of sufficient conditions to observe the convergence to an ESE of the proposed rule.

In order to show the potential of our contributions in the context of DSCNs, we consider a particular scenario of power control in the uplink of a single-cell system in which devices must guarantee a minimum signal to interference plus noise ratio (SINR). Interestingly, we highlight that in this particular scenario, the proposed behavioral rule converges to an ESE independently of the initial state of the network. This result contrasts with the existing literature. For instance, in [1], Altman et al. studied the problem in the general framework of compact sublattices as action sets. Therein, under the assumption that a solution to the problem exists, they established that a simple behavioral rule known in game theory as the best response dynamics (BRD) [4] only converge to the solution from particular starting points. When the transmit power sets are continuous, Yates et al. proved that the BRD converge from any initial point in [14]. In the case of discrete actions sets, an algorithm close to the BRD is proposed in [12]. However, there are still conditions on the starting point to ensure the convergence of the algorithm.

The remainder of this paper unfolds as follows. In Sec. 2, we revisit the notion of satisfaction equilibrium (SE) and ESE and we formulate the QoS provisioning problem in the most general terms. In Sec. 3, we describe our main contribution: a behavioral rule that allows DSCNs to converge to an ESE, when action 
sets correspond to compact sublattices. In Sec. 4, we present numerical results in a particular scenario as described above in order to verify our theoretical contributions. Finally, we conclude our work in Sec. 5.

\section{QoS Provisioning and Games in Satisfaction Form}

\subsection{QoS Problem Formulation}

Consider a DSCN comprising a set $\mathcal{K}=\{1, \ldots, K\}$ of $K$ transmitter $/$ receiver pairs to which we refer as links. Each radio device autonomously chooses its optimal transmit/receive configuration in order to satisfy its own QoS requirements. Here, we denote by $k \in \mathcal{K}$ the $k$-th link, independently of whether it is the transmitter or the receiver that is the device performing the self-adaptation of the link configuration. We denote by $a_{k}$ the transmit/receive configuration adopted by the link $k$, and we denote by $\mathcal{A}_{k}$ the set of all its possible choices. For all $k \in \mathcal{K}, \mathcal{A}_{k}$ is assumed to be a compact sublattice, as in $[1,13] . \mathcal{A}=\mathcal{A}_{1} \times \ldots \times \mathcal{A}_{K}$ represents the set of all configuration profiles. This structure has the advantage of comprising both compact continuous sets and discrete sets ${ }^{1}$. We denote by $\boldsymbol{a}_{-k}=\left(a_{1}, \ldots, a_{k-1}, a_{k+1}, \ldots, a_{K}\right)$ the vector obtained by dropping the $k$-th component of the vector $\boldsymbol{a}$. We denote the space in which the vector $\boldsymbol{a}_{-k}$ exists by $\mathcal{A}_{-k}$. With a slight abuse of notation, we write the vector $\boldsymbol{a}$ as $\left(a_{k}, \boldsymbol{a}_{-k}\right)$, in order to emphasize its $k$-th component. A transmit/receive configuration can be described by parameters such as the power allocation policy, modulation scheme, constellation size, decoding order, scheduling policy, etc. The instantaneous performance of radio device $k$ is determined by a set of $Q_{k}$ functions

$$
\left\{\begin{array}{c}
u_{k}^{(1)}: \mathcal{A} \rightarrow \mathbb{R} \\
\vdots \\
u_{k}^{\left(Q_{k}\right)}: \mathcal{A} \rightarrow \mathbb{R} .
\end{array}\right.
$$

Typical performance metrics are transmission rate, transmission delay, bit error rate, energy efficiency, or any combination of those. We denote the minimum and maximum acceptable values of the performance metric $u_{k}^{\left(q_{k}\right)}$ by $\Gamma_{k}^{\left(q_{k}, \min \right)}$ and $\Gamma_{k}^{\left(q_{k}, \max \right)}$, respectively. Thus, we say that the configuration profile $\boldsymbol{a} \in \mathcal{A}$ satisfies the QoS constrains of the DSCN if for all link $k$ the following set of inequalities are satisfied :

$$
\left\{\begin{array}{c}
\Gamma_{k}^{(1, \min )}<u_{k}^{(1)}\left(a_{k}, \boldsymbol{a}_{-k}\right)<\Gamma_{k}^{(1, \max )} \\
\vdots \\
\Gamma_{k}^{\left(Q_{k}, \min \right)}<u_{k}^{\left(Q_{k}\right)}\left(a_{k}, \boldsymbol{a}_{-k}\right)<\Gamma_{k}^{\left(Q_{k}, \max \right)} .
\end{array}\right.
$$

\footnotetext{
${ }^{1}$ The results of Sec. 3.2 and Sec. 3.4 apply to the general framework of compact sublattices whereas the results of Sec. 3.5 apply only to discrete configuration sets.
} 
Note that the performance metrics of link $k$ depend not only on its own configuration $a_{k}$ but also on the configurations $\boldsymbol{a}_{-k}$ adopted by all the other links. Thus, in order to ease our notation, we define the correspondence $f_{k}: \mathcal{A}_{-k} \rightarrow 2^{\mathcal{A}_{k}}$ that determines all the possible configurations of player $k$ that satisfies its QoS constraints. That is $\forall a_{k} \in \mathcal{A}_{k}$

$$
\begin{aligned}
& a_{k} \in f_{k}\left(\boldsymbol{a}_{-k}\right) \Leftrightarrow \\
& \forall q \in\left\{1, \ldots, Q_{k}\right\}, \Gamma_{k}^{(q, \min )}<u_{k}^{(q)}\left(a_{k}, \boldsymbol{a}_{-k}\right)<\Gamma_{k}^{(q, \max )} .
\end{aligned}
$$

The problem of all the links wanting to satisfy their QoS constraints at the same time can naturally be described as a game.

\subsection{Game Formulation}

As defined in [8], a game in satisfaction form is fully described by the following triplet

$$
\widehat{\mathcal{G}}=\left(\mathcal{K},\left\{\mathcal{A}_{k}\right\}_{k \in \mathcal{K}},\left\{f_{k}\right\}_{k \in \mathcal{K}}\right)
$$

In this triplet, $\mathcal{K}$ represents the set of players, $\mathcal{A}_{k}$ is the strategy set of player $k \in \mathcal{K}$, and the correspondence $f_{k}$ determines the set of actions of player $k$ that allows its satisfaction given the actions played by all the other players. A strategy profile is denoted by vector $\boldsymbol{a}=\left(a_{1}, \ldots, a_{K}\right) \in \mathcal{A}$. In general, an important outcome of a game in satisfaction form is the one where all players are satisfied, that is, an SE. The notion of SE was formulated as a fixed point in [8] as follows:

Definition 1 (Satisfaction Equilibrium). An action profile $\boldsymbol{a}^{+}$is an equilibrium for the game $\widehat{\mathcal{G}}=\left(\mathcal{K},\left\{\mathcal{A}_{k}\right\}_{k \in \mathcal{K}},\left\{f_{k}\right\}_{k \in \mathcal{K}}\right)$ if

$$
\forall k \in \mathcal{K}, \quad a_{k}^{+} \in f_{k}\left(\boldsymbol{a}_{-k}^{+}\right) .
$$

As we shall see, the SE is often not unique and thus, there might exist some SEs that are of particular interest. In the following, we introduce the notion of an efficient SE (ESE). For this intent, we consider a cost function for each player of the game, in order to model the notion of effort or cost associated with a given action choice. For all $k \in \mathcal{K}$, the cost function $c_{k}: \mathcal{A}_{k} \rightarrow[0,1]$ satisfies the following condition : $\forall\left(a_{k}, a_{k}^{\prime}\right) \in \mathcal{A}_{k}^{2}$, it holds that

$$
c_{k}\left(a_{k}\right)<c_{k}\left(a_{k}^{\prime}\right)
$$

if and only if, $a_{k}$ requires a lower effort than action $a_{k}^{\prime}$ when it is played by player $k$. Under the notion of effort, the set of SEs that are of particular interest are those that require the lowest individual efforts. We formalize this notion of equilibrium using the following definition.

Definition 2 (Efficient Satisfaction Equilibrium). An action profile $\boldsymbol{a}^{*}$ is an ESE for the game $\widehat{\mathcal{G}}=\left(\mathcal{K},\left\{\mathcal{A}_{k}\right\}_{k \in \mathcal{K}},\left\{f_{k}\right\}_{k \in \mathcal{K}}\right)$, with cost functions $\left\{c_{k}\right\}_{k \in \mathcal{K}}$, if 


$$
\forall k \in \mathcal{K}, \quad a_{k}^{*} \in f_{k}\left(\boldsymbol{a}_{-k}^{*}\right)
$$

and

$$
\forall k \in \mathcal{K}, \forall a_{k} \in f_{k}\left(\boldsymbol{a}_{-k}^{*}\right), c_{k}\left(a_{k}\right) \geq c_{k}\left(a_{k}^{*}\right) .
$$

Note that the effort associated by each player with each of its actions does not depend on the choice of effort made by other players. Here, we have left players to individually choose their cost functions, which adds another degree of freedom to the modeling of the QoS problem in DSCNs.

Note also that a game in satisfaction form is not a game with a constrained set of actions, as is the case in the formulation presented in [3]. Here, a player can use any of its actions independently of all the other players. The dependency on the other players' actions enters through whether the player under study is satisfied or not.

\subsection{Power Control Game}

In the rest of this paper, we use the context of uplink power control in a singlecell as a case study. Although most of our results apply in a general context, we concentrate in the uplink power control problem as presented in $[12,14]$, to illustrate our results.

Consider $K$ transmitter/receiver pairs denoted by index $k \in \mathcal{K}$. For all $k \in \mathcal{K}$, transmitter $k$ uses power level $p_{k} \in \mathcal{A}_{k}$, with $\mathcal{A}_{k}$ generally defined as a compact sublattice For each player $k \in \mathcal{K}$, we denote $p_{k}^{\min }$ and $p_{k}^{\max }$ the minimum and the maximum power levels in $\mathcal{A}_{k}$, respectively. For every couple $(i, j) \in \mathcal{K}^{2}$, we denote by $g_{i j}$ the channel gain coefficient between transmitter $i$ and receiver $j$. The considered metric for each pair $k$ is the Shannon rate given by

$$
u_{k}\left(p_{k}, \boldsymbol{p}_{-k}\right)=\log _{2}\left(1+\frac{p_{k} g_{k k}}{\sigma_{k}^{2}+\sum_{j \neq k} p_{j} g_{j k}}\right) \quad[\mathrm{bps} / \mathrm{Hz}]
$$

where $\sigma_{k}^{2}$ is the noise variance at receiver $k$.

The QoS requirement for each pair $k$ is to have a channel capacity $u_{k}\left(p_{k}, \boldsymbol{p}_{-k}\right)$ higher than a given threshold $\Gamma_{k}$ bps/Hz. The satisfaction correspondence of link $k$ is then

$$
\begin{aligned}
f_{k}\left(\boldsymbol{p}_{-k}\right) & =\left\{p_{k} \in \mathcal{A}_{k} \mid u_{k}\left(p_{k}, \boldsymbol{p}_{-k}\right) \geq \Gamma_{k}\right\} \\
& =\left\{p_{k} \in \mathcal{A}_{k} \mid p_{k} \geq\left(2^{\Gamma_{k}}-1\right) \frac{\sigma_{k}^{2}+\sum_{j \neq k} p_{j} g_{j k}}{g_{k k}}\right\} .
\end{aligned}
$$

\section{Convergence to an Efficient Satisfaction Equilibrium}

In this section, we provide sufficient conditions for convergence of the BRD and the robust blind response dynamics (RBRD) to an ESE of the game $\widehat{\mathcal{G}}=$ $\left(\mathcal{K},\left\{\mathcal{A}_{k}\right\}_{k \in \mathcal{K}},\left\{f_{k}\right\}_{k \in \mathcal{K}}\right)$, with cost functions $\left\{c_{k}\right\}_{k \in \mathcal{K}}$. 


\subsection{Best Response Dynamics}

In the context of a game in satisfaction form $\widehat{\mathcal{G}}=\left(\mathcal{K},\left\{\mathcal{A}_{k}\right\}_{k \in \mathcal{K}},\left\{f_{k}\right\}_{k \in \mathcal{K}}\right)$, with cost functions $\left\{c_{k}\right\}_{k \in \mathcal{K}}$, we define the best response (BR) correspondence of player $k$, given that the other players adopt the reduced action profile $\boldsymbol{a}_{-k}$, as follows:

$$
B R_{k}\left(\boldsymbol{a}_{-k}\right)=\arg \min _{a_{k} \in f_{k}\left(\boldsymbol{a}_{-k}\right)} c_{k}\left(a_{k}\right) .
$$

We consider a BRD defined as the behavioral rule in which players sequentially update their action following the Gauss-Seidel method [2]. At step $n+1$ of the algorithm, all the players sequentially update their actions with the following rule:

$$
a_{k}^{(n+1)}=B R_{k}\left(a_{1}^{(n+1)}, \ldots, a_{k-1}^{(n+1)}, a_{k+1}^{(n)}, \ldots, a_{K}^{(n)}\right) .
$$

For a discrete set of actions, the BRD can be compared to the asynchronous version of the Minimum Feasible Value Assignment (MFVA) algorithm presented in [12]. The difference is that in [12], players only move to their optimal satisfying action if they are not satisfied with actions played at the previous step. In the BRD, players move to their optimal satisfying action independently of their satisfaction at the previous step.

\subsection{Convergence of the BRD}

To study the convergence of the BRD, we first define some notation of interest. Let $\boldsymbol{a}=\left(a_{1}, \ldots, a_{N}\right)$ and $\boldsymbol{b}=\left(b_{1}, \ldots, b_{N}\right)$ be two action profiles and let $\boldsymbol{c}=$ $\boldsymbol{a} \vee \boldsymbol{b}$ denote the maximum of $(\boldsymbol{a}, \boldsymbol{b})$ component wise, i.e., $\boldsymbol{c}=\left(c_{1}, \ldots, c_{N}\right)$ with $c_{n}=\max \left(a_{n}, b_{n}\right) \forall n \in\{1, \ldots, N\}$. In a similar way, $\boldsymbol{a} \wedge \boldsymbol{b}$ denotes $\min (\boldsymbol{a}, \boldsymbol{b})$ component wise.

Definition 3 (S-modularity). The function $g: \mathcal{A} \rightarrow \mathbb{R}$ is said to be supermodular if for any $\boldsymbol{a}, \boldsymbol{b} \in \mathcal{A}$

$$
g(\boldsymbol{a} \wedge \boldsymbol{b})+g(\boldsymbol{a} \vee \boldsymbol{b}) \geq g(\boldsymbol{a})+g(\boldsymbol{b}),
$$

and said to be submodular if

$$
g(\boldsymbol{a} \wedge \boldsymbol{b})+g(\boldsymbol{a} \vee \boldsymbol{b}) \leq g(\boldsymbol{a})+g(\boldsymbol{b}) .
$$

In the case of the cost function defined in $(6), c_{k}$ depends only on the actions of player $k$. Hence, $c_{k}$ is both supermodular and submodular. As a result, (13) and (14) are equalities.

Definition 4 (Ascending and descending properties). The correspondence $f_{k}$ is said to possess the ascending property (respectively the descending property) if for any two elements $\boldsymbol{a}_{-k}$ and $\boldsymbol{a}_{-k}^{\prime}$ of the set $\mathcal{A}_{-k}$, with $\boldsymbol{a}_{-k}=\boldsymbol{a}_{-k} \wedge \boldsymbol{a}_{-k}^{\prime}$ implies that $\forall a_{k} \in f_{k}\left(\boldsymbol{a}_{-k}\right)$ and $\forall a_{k}^{\prime} \in f_{k}\left(\boldsymbol{a}_{-k}^{\prime}\right)$,

$$
\left\{\begin{array}{l}
\min \left(a_{k}, a_{k}^{\prime}\right) \in f_{k}\left(\boldsymbol{a}_{-k}\right), \\
\max \left(a_{k}, a_{k}^{\prime}\right) \in f_{k}\left(\boldsymbol{a}_{-k}^{\prime}\right),
\end{array}\right.
$$


or for the descending property

$$
\left\{\begin{array}{l}
\max \left(a_{k}, a_{k}^{\prime}\right) \in f_{k}\left(\boldsymbol{a}_{-k}\right), \\
\min \left(a_{k}, a_{k}^{\prime}\right) \in f_{k}\left(\boldsymbol{a}_{-k}^{\prime}\right) .
\end{array}\right.
$$

An important consequence of the ascending (or descending) property is that

$$
\forall \boldsymbol{a}_{-k} \in \mathcal{A}_{-k}, f_{k}\left(\boldsymbol{a}_{-k}\right) \neq \varnothing
$$

The definition of an ascending set can easily be understood in the context of distributed power control. In such a context, the ascending property means that if all the other players increase their powers, player $k$ also has to increase its own power if it wants to remain satisfied. Also note that if the ascending property is ensured, then there is always at least one satisfying power level for player $k$, whatever the other players are playing. In particular, when all the players are at maximum power levels, there exists a satisfying power for player $k$, which is a strong assumption.

Proposition 1. Assume that for all $k \in \mathcal{K}, f_{k}(\cdot)$ is nonempty and compact for all the values of their arguments, $f_{k}(\cdot)$ has either the ascending or the descending property and $f_{k}(\cdot)$ is continuous. Then the following holds:

- (i) An ESE exists.

- (ii) If the dynamics start with the action profile associated with the highest or lowest effort in $c_{k}(\cdot)$, for all $k \in \mathcal{K}$, the BRD converge monotonically to an ESE.

- (iii) If the dynamics start from an SE, the trajectory of the best response converges to an ESE. It monotonically evolves in all components.

- (iv) In a two-player game, the BRD converge to an ESE from any starting point.

The proof of Prop. 1 comes from Th. 1 in [1] and Th. 2.3 in [13]. We simply have to verify that the right assumptions hold for the ascending case and the descending case:

- Let $f_{k}(\cdot)$ be ascending for all $k \in \mathcal{K} . c_{k}$ is a cost function player $k$ wants to minimize, in particular $c_{k}$ is a submodular function, and thus $-c_{k}$ is a supermodular function player $k$ wants to maximize and Th. 1 from [1] holds, i.e., (i, ii, iii) in Prop. 1 are ensured when the sets are ascending.

- Let $f_{k}(\cdot)$ be descending for all $k \in \mathcal{K}$. A similar reasoning can be made: $c_{k}$ is a submodular function player $k$ wants to minimize and the same theorem holds as well, i.e., (i, ii, iii) in Prop. 1 are ensured when the sets are descending.

In both ascending and descending cases, (iv) in Prop. 1 is obtained from Th. 2.3 in $[13]$. 


\subsection{BRD in the Uplink Power Control Game}

In the general framework of compact sublattices as strategy sets (including continuous and discrete action sets), the BRD converge only from given starting points (see [1, 13]). However, in the uplink power control problem, it has been shown in $[10,14]$ that when strategy sets are continuous, the BRD converge from any initial point. When strategy sets are discrete, the convergence of the BRD from any initial point to an equilibrium is not guaranteed. In [12], it is shown that the MFVA converges only when all the transmitters start at their lowest power levels. In the following, we consider a 3-player uplink power control game to illustrate the non-convergence of the BRD from a particular initial action profile.

Example 1. In this example, we refer to the notation introduced in Sec. 2.3. Let us consider $K=3$ pairs of transmitters/receivers. For all $k \in \mathcal{K}$, transmitter $k$ uses power level $a_{k} \in\left\{p^{\min }, p^{\max }\right\}$. Given the constraints from Sec. 2.3, let consider channel gains such that

$$
\begin{aligned}
& f_{1}\left(p^{\min }, p^{\min }\right)=f_{3}\left(p^{\min }, p^{\min }\right)=\left\{p^{\min }, p^{\max }\right\} \\
& f_{1}\left(p^{\min }, p^{\max }\right)=f_{3}\left(p^{\min }, p^{\max }\right)=\left\{p^{\min }, p^{\max }\right\} \\
& f_{1}\left(p^{\max }, p^{\min }\right)=f_{3}\left(p^{\max }, p^{\min }\right)=\left\{p^{\max }\right\} \\
& f_{1}\left(p^{\max }, p^{\max }\right)=f_{3}\left(p^{\max }, p^{\max }\right)=\left\{p^{\max }\right\}
\end{aligned}
$$

and

$$
\begin{aligned}
& f_{2}\left(p^{\min }, p^{\min }\right)=\left\{p^{\min }, p^{\max }\right\} \\
& f_{2}\left(p^{\min }, p^{\max }\right)=\left\{p^{\max }\right\} \\
& f_{2}\left(p^{\max }, p^{\min }\right)=\left\{p^{\min }, p^{\max }\right\} \\
& f_{2}\left(p^{\max }, p^{\max }\right)=\left\{p^{\max }\right\}
\end{aligned}
$$

We can check that $f_{k}$ has the ascending property for all $k \in \mathcal{K}$. For each pair $k$, the cost of the power level is given by the identity cost function $c_{k}\left(a_{k}\right)=a_{k}$. This game has two ESEs:

- $\left(p^{\min }, p^{\min }, p^{\min }\right)$ where all the players transmit at their lowest power level. No player has interest in deviating from its action since any other action has a higher cost (even though the player would remain satisfied).

- $\left(p^{\max }, p^{\max }, p^{\max }\right)$ where all the players have to transmit at maximum power to be satisfied. If one deviates from its action, it will not be satisfied anymore.

But depending on the initial action profile of the BRD, the BRD may not converge to an ESE. For instance, assume that the BRD start at $\boldsymbol{p}^{(0)}=$ $\left(p^{\max }, p^{\min }, p^{\max }\right)$. At step 1, player 1 chooses the action that minimizes $c_{1}(\cdot)$ given the previous actions of the other players $\boldsymbol{p}_{-1}^{(0)}=\left(p^{\min }, p^{\max }\right)$, i.e.,

$$
p_{1}^{(1)}=B R_{1}\left(p^{\min }, p^{\max }\right)=p^{\min } .
$$

Player 2 chooses the action that minimizes $c_{2}(\cdot)$ given the most recent actions of the other players $\left(p_{1}^{(1)}, \boldsymbol{p}_{-(1,2)}^{(0)}\right)=\left(p^{\min }, p^{\max }\right)$, i.e., 


$$
p_{2}^{(1)}=B R_{2}\left(p^{\min }, p^{\max }\right)=p^{\max } .
$$

Player 3 chooses the action that minimizes $c_{3}(\cdot)$ given $\left(p_{1}^{(1)}, p_{2}^{(1)}\right)=\left(p^{\min }, p^{\max }\right)$, i.e.,

$$
p_{3}^{(1)}=B R_{3}\left(p^{\min }, p^{\max }\right)=p^{\min } .
$$

At step 2, player 1 chooses the action that minimizes $c_{1}(\cdot)$ given the previous actions of the other players $\boldsymbol{p}_{-1}^{(1)}=\left(p^{\max }, p^{\min }\right)$, i.e.,

$$
p_{1}^{(2)}=B R_{1}\left(p^{\max }, p^{\min }\right)=p^{\max } .
$$

Player 2 chooses the action that minimizes $c_{2}(\cdot)$ given the most recent actions of the other players $\left(p_{1}^{(2)}, \boldsymbol{p}_{-(1,2)}^{(1)}\right)=\left(p^{\max }, p^{\min }\right)$, i.e.,

$$
p_{2}^{(2)}=B R_{2}\left(p^{\max }, p^{\min }\right)=p^{\min } .
$$

Player 3 chooses the action that minimizes $c_{3}(\cdot)$ given $\left(p_{1}^{(2)}, p_{2}^{(2)}\right)=\left(p^{\max }, p^{\min }\right)$, i.e.,

$$
p_{3}^{(2)}=B R_{3}\left(p^{\max }, p^{\min }\right)=p^{\max } .
$$

The algorithm is back at the starting point, and it is clear that it will continue in this infinite loop.

\subsection{Robust Blind Response Dynamics}

The BRD have significant drawbacks. First, it was just shown that in a $K$-player game with $K>2$, the dynamics may not converge to an ESE depending on the initial action profile. Second, to determine the BR, each player has to know the set $f_{k}\left(\boldsymbol{a}_{-k}\right) \forall \boldsymbol{a}_{-k} \in \mathcal{A}_{-k}$. To overcome these drawbacks, we propose a new algorithm that requires less information about the game for each player and can still be proven to converge to an ESE. Let us start by defining the robust blind response (RBR) by $R B R_{k}: \mathcal{A} \rightarrow \mathcal{A}_{k}$, such that :

$$
\left(a_{k}, \boldsymbol{a}_{-k}\right) \rightarrow\left\{\begin{array}{l}
a_{k}^{\prime}, \text { if } a_{k}^{\prime} \in f_{k}\left(\boldsymbol{a}_{-k}\right), a_{k} \in f_{k}\left(\boldsymbol{a}_{-k}\right) \text { and } c_{k}\left(a_{k}^{\prime}\right) \leq c_{k}\left(a_{k}\right), \\
a_{k}^{\prime}, \text { if } a_{k}^{\prime} \in f_{k}\left(\boldsymbol{a}_{-k}\right) \text { and } a_{k} \notin f_{k}\left(\boldsymbol{a}_{-k}\right), \\
a_{k}, \text { otherwise, }
\end{array}\right.
$$

with action $a_{k}^{\prime}$ being randomly chosen in $\mathcal{A}_{k}$, such that $\forall a_{k} \in \mathcal{A}_{k}, \operatorname{Pr}\left(a_{k}^{\prime}=a_{k}\right)>$ 0 . Each time the RBR is used, a player $k \in \mathcal{K}$ randomly chooses an action in its strategy set $\mathcal{A}_{k}$ without taking into account the constraints of other players. Player $k$ only has to know if the new action and the previous one allow the satisfaction of its individual constrains and to compare their respective costs. If both actions allow the satisfaction of the constraints, it chooses the one with the lowest cost. If the new action allows the satisfaction of the individual constraints whereas the previous one does not, it moves to the new action. Otherwise, it keeps the same action. When all the players sequentially use the RBR such that $\forall k \in \mathcal{K}$ 


$$
a_{k}^{(n+1)}=R B R_{k}\left(a_{1}^{(n+1)}, \ldots, a_{k-1}^{(n+1)}, a_{k+1}^{(n)}, \ldots, a_{K}^{(n)}\right),
$$

we refer to these dynamics as the RBR dynamics (RBRD). Our main result in this section is stated in the following theorem.

Theorem 1. Assume that for all $k \in \mathcal{K}, f_{k}(\cdot)$ is nonempty and compact for all the values of their arguments, $f_{k}(\cdot)$ has the ascending property and it is continuous, and $c_{k}(\cdot)$ is strictly increasing. Then, the following holds:

- (i) If the dynamics start from an SE, the sequence of RBRs converges to an ESE. It monotonically decreases in all components.

- (ii) If the dynamics start with the actions associated with the highest effort in $c_{k}(\cdot), \forall k \in \mathcal{K}$, the sequence of RBRs converges monotonically to an ESE.

- (iii) In a two-player game, the sequence of RBRs converge to an ESE from any starting point.

Proof. Applying Prop. 1, we know that there exists an ESE for the game $\widehat{\mathcal{G}}=$ $\left(\mathcal{K},\left\{\mathcal{A}_{k}\right\}_{k \in \mathcal{K}},\left\{f_{k}\right\}_{k \in \mathcal{K}}\right)$. The convergence of the RBRD to an ESE is proven in two steps. First, we show for (i, ii, ii) that the RBRD converge to a fixed point. Second, we explain why this fixed point has to be an ESE.

- (i) Assume that the dynamics start from an SE: $\boldsymbol{a}^{S E}$ and this SE is not an ESE (otherwise, the convergence is trivial). Let player $k \in \mathcal{K}$ be the first player to actually change its action at step $n$ to $a_{k}^{(n)}$; necessarily this action has a lower cost than $a_{k}^{S E}$ because a satisfied player can only move to another satisfying action with a lower cost. Let the next player to move be denoted by $j$. From its point of view $\left(a_{k}^{(n)}, \boldsymbol{a}_{-\{k, j\}}^{S E}\right)=\left(a_{k}^{(n)}, \boldsymbol{a}_{-\{k, j\}}^{S E}\right) \wedge \boldsymbol{a}_{-j}^{S E}$. Hence, due to the ascending property of $f_{j}$ and the strict monotony of $c_{j}$, necessarily its new action $a_{j}^{\left(n^{\prime}\right)} \leq a_{j}^{S E}$, and so forth. For each $k \in \mathcal{K}$ the sequence $\left\{a_{k}^{(n)}\right\}_{n \in \mathbb{N}}$ is decreasing in a compact set. Thus, the algorithm converges to a limit.

- (ii) Assume that the dynamics start from action profile $\boldsymbol{a}^{\max }=\left(a_{1}^{\max }, \ldots, a_{K}^{\max }\right)$ and this point is not an SE (otherwise refer to (i)). Let player $k$ update its action first, at step $n$. Necessarily, its updated action $a_{k}^{(n)}$ is lower than $a_{k}^{\max }$. Then $\forall j \neq k, j \in \mathcal{K}$

$$
\left(\boldsymbol{a}_{\{-j, k\}}^{\max }, a_{k}^{(n)}\right)=\left(\boldsymbol{a}_{\{-j, k\}}^{\max }, a_{k}^{(n)}\right) \wedge \boldsymbol{a}_{-j}^{\max } .
$$

Due to the ascending property of $f_{j}$ and the strict monotony of $c_{j}$, the update of player $j$ is hence lower than $a_{j}^{\max }$, and so forth. Again, for each player $k \in \mathcal{K}$, the sequence of action $\left\{a_{k}^{(n)}\right\}_{n \in \mathbb{N}}$ is decreasing in a compact set and the algorithm converges to a limit.

- (iii) In a two-player game, assume the dynamics start from a random action profile $\left(a_{1}^{(0)}, a_{2}^{(0)}\right)$. Assume player 1 is the first player that updates its action to get satisfied, at step $n$. The action profile is then $\left(a_{1}^{(n)}, a_{2}^{(0)}\right)$. In the next move, either the same player 1 decreases its action, remaining satisfied, or player 2 moves to an action that satisfies it, leading to an action profile $\left(a_{1}^{(n)}, a_{2}^{\left(n^{\prime}\right)}\right)$. If this profile is an $\mathrm{SE}$, the dynamics converge according to (i). Otherwise 
player 1 is no longer satisfied and has to update its action. If $a_{2}^{\left(n^{\prime}\right)}<a_{2}^{(0)}$, then due to the ascending property and the strict monotonicity of $c_{1}$, player 1 will only move to a lower action than $a_{1}^{(n)}$. Then player 2 will also have to move to a lower action than $a_{2}^{\left(n^{\prime}\right)}$ for analogous reasons, and so forth. The sequences $\left\{a_{1}^{(n)}\right\}_{n \in \mathbb{N}}$ and $\left\{a_{2}^{(n)}\right\}_{n \in \mathbb{N}}$ are hence decreasing in a compact set, so they converge to a limit. If $a_{2}^{\left(n^{\prime}\right)}>a_{2}^{(0)}$, the sequences are increasing in a compact set and converge as well.

We now have to prove that a fixed point is an ESE. Consider that $\boldsymbol{a}^{*}$ is a fixed point for $R B R_{k}, \forall k \in \mathcal{K}$. By the definition of $R B R_{k}$ this means that there exists no $a_{k} \in \mathcal{A}_{k}$ such that $a_{k} \in f_{k}\left(\boldsymbol{a}_{-k}^{*}\right)$ and $c_{k}\left(a_{k}\right) \leq c_{k}\left(a_{k}^{*}\right)$, which is exactly the definition of the ESE. This completes the proof.

The main advantage of these dynamics over BRD in a general framework is that the former require only local information and the knowledge of an explicit expression for $f_{k}$ is no longer relevant. Only the knowledge of whether the corresponding player is satisfied or not is sufficient to implement the RBR.

\subsection{RBRD in the Uplink Power Control Game}

A very interesting property occurs for the RBR in the uplink power control game with discrete action sets.

Theorem 2. In the power allocation game defined above in Sec. 2.3, with discrete action sets, i.e., $\forall k \in \mathcal{K}, \mathcal{A}_{k}=\left\{p_{k}^{(1)}, \ldots, p_{k}^{N_{k}}\right\}$ with $N_{k}$ the number of power levels in action set $\mathcal{A}_{k}$, the RBRD converge to an ESE from any starting point.

Proof. We show in this proof that from any starting point of the dynamics, there is a non-null probability that the dynamics move to a particular SE with a given way. Note that the particular sequence of events we describe here is not always the way the dynamics run. It is simply a sequence that can occur with a non-null probability, but there are many other possible sequences that lead to an SE.

Assume $\boldsymbol{p}^{(0)}=\left(p_{1}^{(0)}, \ldots, p_{k}^{(0)}\right)$ is the starting power profile of the dynamics. Consider all the unsatisfied players at this point and assume that they all move to their maximum possible power levels (this may happen with a non-null probability). These levels satisfy them since the ascending property gives us

$$
\forall k \in \mathcal{K}, \forall \boldsymbol{p}_{-k} \in \mathcal{A}_{-k}, p_{k}^{\max } \in f_{k}\left(\boldsymbol{p}_{-k}\right) .
$$

This increase of power levels may cause some of the satisfied players at the starting point not to be satisfied anymore. We also assume that these players move to their maximum power levels. And the same is done until no unsatisfied player remains. So we get a power profile made of the highest power levels for some of the players and the initial power levels for the others, and every player is satisfied at this point: it is an SE.

Finally, from (i) of Th. 1, the dynamics converge to an ESE, which completes the proof. 
Th. 2 highlights a very interesting property of the RBRD when players enter or quit the game (or when the channel coefficients vary). Indeed, if $K$ transmitters are in any given ESE $\boldsymbol{p}^{*}$ and a new transmitter enters the game, a new game starts with $K+1$ players. Thus, from Th. 2 , it can be stated that convergence to a new ESE, if it exists, is ensured from the new starting point $\left(\boldsymbol{p}^{*}, p_{k+1}\right)$.

\section{Numerical Results}

In this section, we provide numerical results for the uplink power control game with discrete action sets as defined in Sec. 2.3.

In Fig. 1, we show the sequences of actions converging to an ESE for the RBRD in a 2-player power control game. The colored region is the satisfaction region, i.e., the region allowing both players to be satisfied. The coloring of this region follows the sum of the costs for each player. The RBR first converges to the satisfaction region, then converges to an ESE while remaining in the satisfaction region.

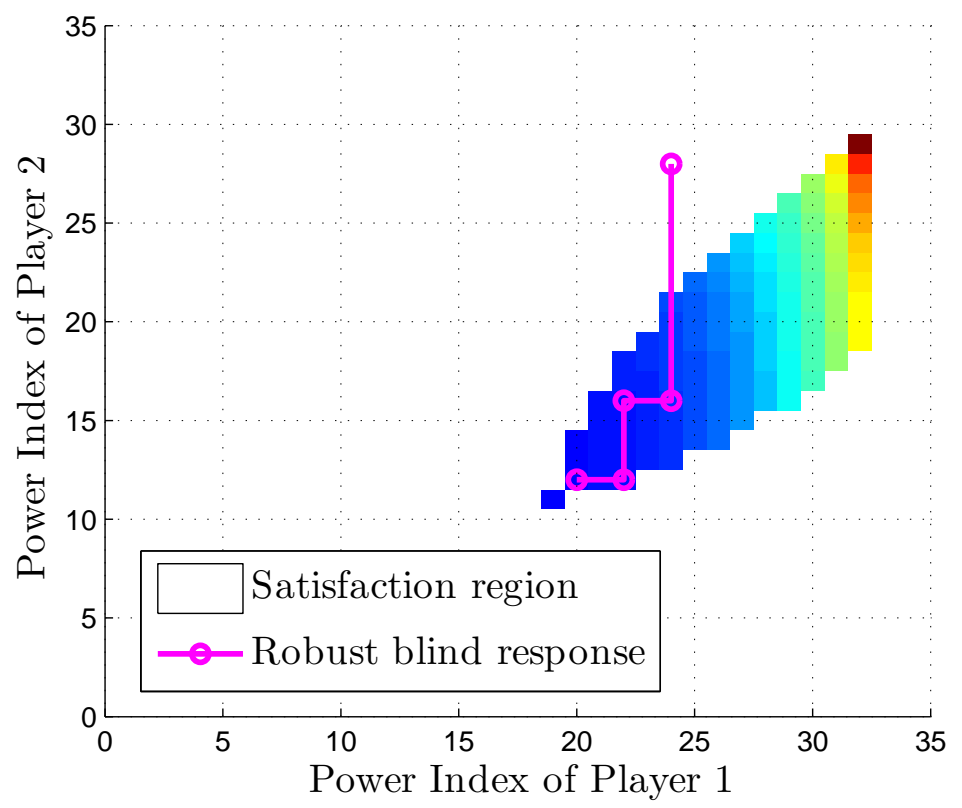

Fig. 1. Sequence of power indices for the RBRD in the uplink 2-player power control game. The colored region is the satisfaction region, i.e., the region where the two players mutually satisfy their constraints.

The scenario we consider in Fig. 2 and Fig. 3 highlights the advantages of RBRD over the BRD in a 3-player game: during the first 200 steps, only 
transmitters 1 and 3 are in the game, then transmitter 2 joins them for the 200 next steps, and finally transmitter 3 leaves for the last 200 steps. On each of the two figures, we show the sequence of power indices for the three players, knowing that each action set is made of $N_{k}=32$ possible power levels from $10^{-6} \mathrm{~W}$ to $10^{-2} \mathrm{~W}$. We also show the satisfaction states of the three players: for each step of the dynamics, if all the player are satisfied, the satisfaction state is 1 , otherwise it is 0. Fig. 2 and Fig. 3 correspond to the behavior of the BRD and the RBRD, respectively. The channel parameters and the starting points of the two simulations are exactly the same. Channel gains are $g_{22}=10^{-5}$, $g_{11}=g_{33}=g_{13}=g_{21}=g_{32}=10^{-6}, g_{12}=g_{23}=g 31=10^{-7}$, and transmitters 1,2 , and 3 start at power levels $10^{-3} \mathrm{~W}, 10^{-5 / 2} \mathrm{~W}$, and $10^{-9 / 4} \mathrm{~W}$, respectively. The utility constraints $\Gamma_{1}, \Gamma_{2}$, and $\Gamma_{3}$ are taken as $1.2 \mathrm{bps} / \mathrm{Hz}, 1.5 \mathrm{bps} / \mathrm{Hz}$, and $1.2 \mathrm{bps} / \mathrm{Hz}$, respectively. The variance of the noise is fixed at $10^{-10} \mathrm{~W}$ for all the transmitters. It is interesting to notice that the BRD converge to ESE during the first and third phase but when transmitter 2 enters the game in the second phase, the BRD do not converge to an ESE. Instead, they enter a loop and we can see that the transmitters are not satisfied. Concerning the RBRD, although their convergence time is longer, they converge in the three phases and another interesting fact is that transmitters are satisfied during a longer amount of time compared to the BRD.
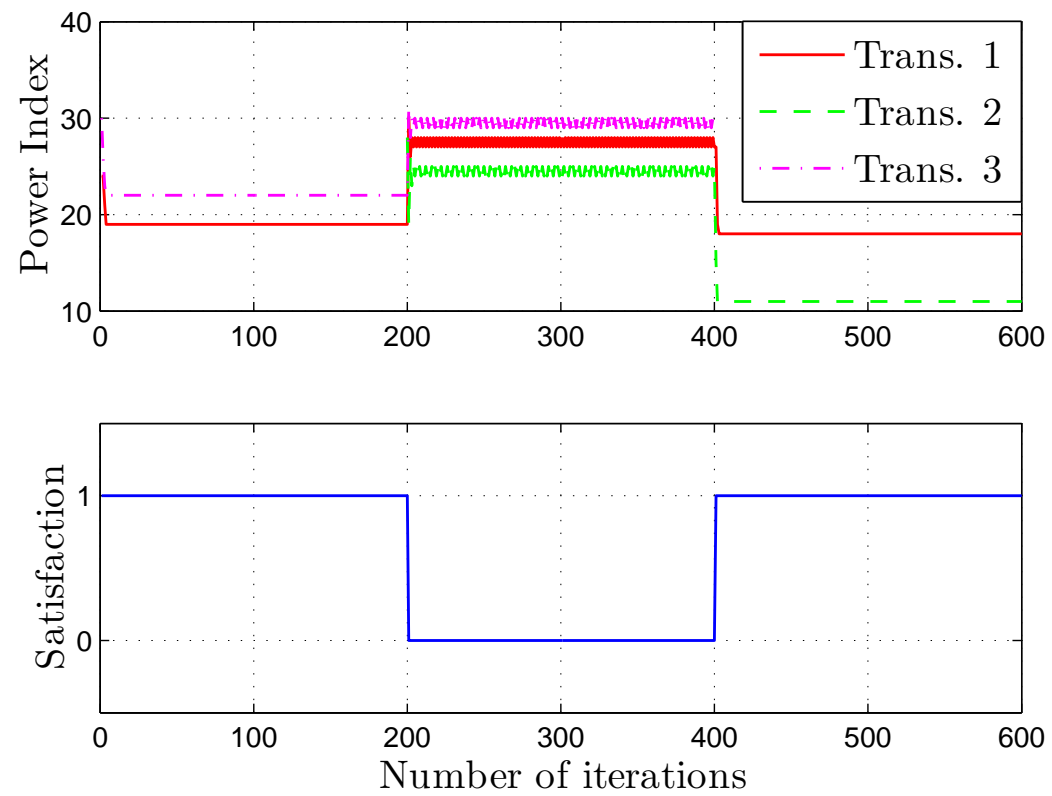

Fig. 2. Sequences of power indices and satisfaction states for the BRD in the 3-player uplink power control game. 

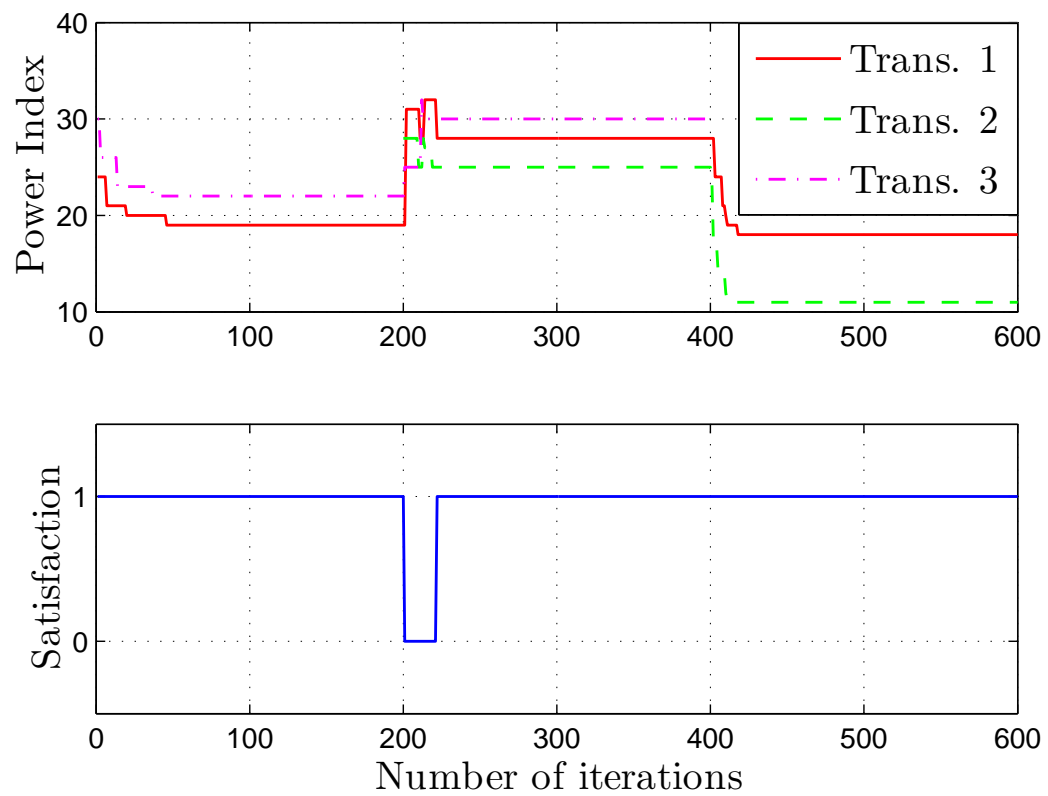

Fig. 3. Sequences of power indices and satisfaction states for the RBRD in the 3-player uplink power control game.

\section{Conclusion and Future Work}

In this work, we have proposed a behavior rule that converges to an ESE in the general framework of compact sublattices as actions sets. Compared to the BRD, the proposed rule requires far less information although its convergence time is longer. Applying this rule to the uplink power control game with discrete actions sets has been shown to be of great interest since the dynamics are proven to converge to an ESE from any starting action profile. This particular feature allows the proposed rule to be robust to the entrance or the exit of players in the power control game.

However, a strong assumption of this work is to assume that for every player, for any action profile of the other players, there exists an action satisfying the considered player. In the power control game, it would be more relevant to take into account scenarios in which the power levels of the other players are too high and a given player cannot be satisfied for any action it can play. Hence, a natural perspective of this work is to relax this assumption and study the convergence of the dynamics in this context. 


\section{References}

1. E. Altman and Z. Altman. S-modular games and power control in wireless networks. IEEE Transactions on Automatic Control, 48(5):839-842, May 2003.

2. D. P. Bertsekas and J. N. Tsitsiklis. Parallel and Distributed Computation: Numerical Methods. Prentice-Hall, Inc., Upper Saddle River, NJ, USA, 1989.

3. G. Debreu. A social equilibrium existence theorem. Proceedings of the National Academy of Sciences of the United States of America, 38(10):886-893, October 1952.

4. D. Fudenberg and J. Tirole. Game Theory. MIT Press, Cambridge, MA, 1991.

5. Z. Han, D. Niyato, W. Saad, T. Basar, and A. Hjorungnes. Game Theory in Wireless and Communication Networks: Theory, Models and Applications. Cambridge University Press, Cambridge, UK, 2011.

6. S. Haykin. Cognitive radio: Brain-empowered wireless communications. IEEE Journal on Selected Areas in Communications, 23(2):201-220, 2005.

7. S. Lasaulce and H. Tembine. Game Theory and Learning in Wireless Networks: Fundamentals and Applications. Elsevier Academic Press, Waltham, MA, 2011.

8. S. M. Perlaza, H. Tembine, S. Lasaulce, and M. Debbah. Quality-of-service provisioning in decentralized networks: A satisfaction equilibrium approach. IEEE Journal of Selected Topics in Signal Processing, 6(2):104-116, Apr. 2012.

9. L. Rose, S. Lasaulce, S.M. Perlaza, and M. Debbah. Learning equilibria with partial information in decentralized wireless networks. IEEE Communications Magazine, 49(8):136 -142, Aug. 2011.

10. G. Scutari, S. Barbarossa, and D.P. Palomar. Potential games: A framework for vector power control problems with coupled constraints. In the IEEE International Conference on Acoustics, Speech and Signal Processing (ICASSP), Toulouse, France, May 2006.

11. G. Scutari, D. P. Palomar, F. Facchinei, and J.-S. Pang. Convex optimization, game theory, and variational inequality theory in multiuser communication systems. IEEE Signal Processing Magazine, 27(3):35-49, May 2010.

12. C. C. Wu and D. P. Bertsekas. Distributed power control algorithms for wireless networks. IEEE Transactions on Vehicular Technology, 50(2):504-514, Mar. 2001.

13. D. D. Yao. S-modular games, with queueing applications. Queueing Systems, 21(3-4):449-475, 1995.

14. R. D. Yates. A framework for uplink power control in cellular radio systems. IEEE Journal on Selected Areas in Communications, 13(7):1341-1347, Sep. 1995. 